\title{
"Doctor, will that x-ray harm my unborn child?"
}

\author{
Savithiri Ratnapalan MD, Yedidia Bentur MD, Gideon Koren MD
}

Abstract: Exposure to ionizing radiation can be a source of anxiety for many pregnant women and their health care providers. An awareness of the radiation doses delivered by different techniques and the acceptable exposure thresholds can help both patients and practitioners. We describe exposure to radiodiagnostic procedures during pregnancy and suggest an approach to assess the potential risk.

Case 1: A 29-year-old patient underwent an upper gastrointestinal series as part of the diagnosis of prolonged heartburn. She comes to see you 1 week later and is very upset because her period is 1 week late and she was about 4 weeks pregnant at the time of the procedure. The patient asks you to schedule the termination of her pregnancy on the advice of several family members.

Case 2: A 40-year-old woman arrives at the emergency department with acute pleuritic chest pain and shortness of breath. The patient is 15 weeks' pregnant. To rule out a pulmonary embolus, should you perform a ventilationperfusion scan or computed tomography (CT) angiography? Case 3: A 37-year-old woman who is 20-weeks pregnant reports persistent pain in her right upper thigh that is exacerbated after jogging. The pain is localized and has no radicular properties. A physical examination shows localized tenderness on the right hip joint without any abnormal neurological findings. You suspect hip bursitis but want to order a radiograph to rule out osteoarthritis with degenerative changes. The patient is nervous about the possible effects of the radiation on her baby; how would you counsel her?

Many women are exposed to radiation from diagnostic imaging procedures before they know they are pregnant or because it is necessary during a known pregnancy. These patients often question the potential effects of the radiation on the developing fetus, and they may perceive radiation as being very harmful. ${ }^{1-4}$ A realistic and informed approach to counselling these patients can minimize the anxiety felt by both patients and health care providers.

Humans are exposed to both background and man-made sources of radiation. For the purpose of this review, "radiation" refers to ionizing radiation (e.g., x-rays, $\gamma$-rays, radionuclides) and not to other forms of radiation (e.g., long- wavelength electromagnetic waves such as radar, microwaves, diathermy and FM radio waves).

Ionizing radiation in the form of $\mathrm{x}$-rays and $\gamma$-rays are shortwavelength electromagnetic rays. Low-energy photons in X-rays and high-energy photons in $\gamma$-rays can alter the normal structure of a living cell both directly and indirectly. The direct mechanism involves disruption of the atom's structure to produce an ionized compound and a free electron. The indirect mechanism involves radiolysis of water and generation of free radicals. ${ }^{5}$

Ionizing radiation can cause two types of effects. ${ }^{5}$ First, loss of tissue function (deterministic effect) can occur. This type of injury has tissue-specific thresholds and may involve various repair and compensatory mechanisms. If the radiation dose is fractionated, there is greater repair and proliferation, hence there is greater tolerance of the tissue to the radiation. Second, damage can occur from a single random modification in a cell component (e.g., DNA) (stochastic effect). There is no dose threshold for stochastic effects.

Since invention of the x-ray in 1895, ionizing radiation has been harnessed for diagnostic and therapeutic purposes. With the atomic bombings in World War II, the world became aware of the serious potential carcinogenic, teratogenic and mutagenic effects of ionizing radiation. Despite the increase in concern about the health effects of ionizing radiation, the medical use of x-rays has continued to grow. In 1980, the number of radiographs performed in the United States was 225 million, including about 80 million fertile men and women. ${ }^{5}$ In 2006, the estimated total number of radiographs in the US was about 330 million. ${ }^{6}$ The fetus is exposed to unavoidable (background) radiation from cosmic rays, terrestrial radiation from ground and building and naturally occurring radioisotopes that are inhaled or ingested. The total fetal dose from background radiation sources is $0.1 \mathrm{rad}$ or less during the entire pregnancy. ${ }^{5}$

\section{Radiation effects}

High levels of acute exposure to radiation (as low as $100 \mathrm{rad}$, usually above $400 \mathrm{rad}$ ) can cause acute radiation syndrome and even death. Ionizing radiation has several biological effects on reproduction. ${ }^{5}$

Motherisk Program, Department of Pediatrics (Ratnapalan, Koren), Hospi tal for Sick Children, Toronto, Ont.; and the Israel Poison Information Center (Bentur), Rambam Health Care Campus, The Rappaport Faculty of Medicine, Technion-Israel Institute of Technology, Haifa, Israel 


\section{Key points}

- Exposure to diagnostic radiation during pregnancy is associated with high levels of anxiety among pregnant women and their health care providers

- This anxiety may lead women to consider terminating an otherwise wanted pregnancy.

- The vast majority of diagnostic radiation procedures expose the fetus to radiation levels far below the teratogenic range.

- Physicians should use evidence-based counselling to allay misperceptions of risk.

- Irradiation of the testes and ovaries can cause infertility. This can be either temporary or permanent depending on the dose (as low as $15 \mathrm{rad}$ ) and duration of the radiation.

- High-dose (hundreds of rads) abdominal or pelvic irradiation (e.g., for treatment of Wilms tumour) may cause somatic damage to abdominopelvic structure and interfere with conception and gestation.

- At present, there has been no correlation shown between exposure to ionizing radiation in utero and genetic disorders.

- The fundemental effects of ionizing radiation on the developing fetus are intrauterine growth retardation and defects in the central nervous system (microcephaly, mental retardation). The most vulnerable period is 8-15 weeks' gestation. This effect is associated with radiation doses above 10-20 rads.

- Low risk of tumour cannot be ruled out after in utero exposure to less than $10 \mathrm{rad}$. In utero irradiation is not considered likely to significantly increase the lifetime risk of tumour development in a person who lives to old age and who receives additional radiation throughout his or her life.

The dose of the ionizing radiation required to cause specific developmental effects depends on the stage of gestation (Table 1). When assessing radiation exposure during pregnancy, it is important to tally the cumulative dose delivered to the patient. Although a single procedure is usually not associated with reproductive risk, this may not be the case for multiple procedures (e.g., radiographs received by trauma patients).

\section{Dose limits}

Exposure to a cumulative dose of less than 5 rads during pregnancy has not been shown to affect the outcome of the pregnancy compared to control populations exposed to background radiation estimated as less than $0.1 \mathrm{rad}$ over 9 months. ${ }^{2,5,7}$ According to the US National Council on Radiation Protection, fetal risk of malformation increases above background levels at radiation doses above 15 rads.

The US National Council on Radiation Protection states that the risk of induced miscarriages or major congenital malformations in embryos or fetuses exposed to doses of 5 rads or less is negligible compared to the spontaneous risk among nonexposed women. ${ }^{8}$ Spontaneous risk includes a $15 \%$ chance of having a spontaneous abortion, 3\% risk for major malformation and $4 \%$ risk of fetal growth restriction., ${ }^{5,7,10}$ The Radiation Safety Committee of the US Center for Disease Control and Prevention recommends that unborn babies of laboratory workers should not be exposed to more than 0.5 rad cumulatively from all sources of radiation during the entire gestational period. ${ }^{8}$ Typically, occupational radiation exposure is measured by tags or dosimeter badge. At present, no such devices are required for pregnant women undergoing diagnostic radiation.

\section{Estimated exposure}

Most radiodiagnostic examinations result in less than 5 rads radiation to the fetus. ${ }^{2,3,5,10}$ An estimate of radiation doses used for diagnostic imaging procedures can be obtained from most radiology departments (Table 2). It is estimated that about $85 \%$ of a person's lifetime exposure to radiation will come from natural sources (background radiation) and about $15 \%$ will come from man-made sources. ${ }^{11}$ The majority of manmade radiation will result from diagnostic radiology devices (about $97 \%$ from CT scanners).

Direct exposure of a fetus to radiation occurs when the fetus is located within the field being imaged. Indirect exposure is due to scattered radiation from maternal tissues. The fetal dose depends on the radiation dose delivered and the dis-

Table 1: Effects of radiation exposure on prenatal development ${ }^{1}$

\begin{tabular}{|c|c|c|c|}
\hline Gestational stage & $\begin{array}{l}\text { Time after } \\
\text { conception }\end{array}$ & Fetal dose, rad & Potential effect \\
\hline Pre-implantation & $0-14 d$ & $5-10$ & - Prenatal death \\
\hline \multirow[t]{2}{*}{ Major organogenesis } & $1-8 w k$ & $20-25$ & - Growth retardation \\
\hline & $2-15 w k$ & $20-25$ & $\begin{array}{l}\text { - Small head size } \\
\text { - Exposure before } 8 \text { wk does not cause an } \\
\text { intellectual deficit despite small head size } \\
\text { - Most sensitive period for induction of childhood } \\
\text { cancer }\end{array}$ \\
\hline $\begin{array}{l}\text { Rapid neuron development } \\
\text { and migration }\end{array}$ & $6-15 w k$ & $>10$ & $\begin{array}{l}\text { - Small head size, seizures, decline in intelligence } \\
\text { quotient ( } 25 \text { points per } 100 \text { rad) }\end{array}$ \\
\hline \multirow{2}{*}{$\begin{array}{l}\text { After organogenesis and rapid } \\
\text { neuron development }\end{array}$} & $15 \mathrm{wk}$ to term & $>10$ & - Increased frequency of childhood cancer \\
\hline & 15 wk to term & $>50$ & - Severe mental retardation (16-25 wk) \\
\hline
\end{tabular}


tance between the fetus and the area being imaged. Radiation exposure dose is inversely related to the distance (to the power of two) from the radiation source. A lead shield may reduce indirect exposure, but internal scatter in the mother will allow some radiation to reach the fetus. ${ }^{12}$ If thinly layered bismuth radioprotective latex or leaded garments are used to shield the mother's radiosensitive organs, the radiation dose that reaches the fetus can be cut by $50 \% .^{13}$

Fetal doses resulting from radiological examination of the mother's skull, head, neck, chest and extremities are extremely low $(<0.01 \mathrm{rad})$ because of the relatively low maternal radiation dose, beam direction and distance between the primary field and the fetus. ${ }^{1}$ A recent study claiming an association between dental radiography in pregnancy and low birth weight ${ }^{14}$ has been criticized for lack of biological plausibility. ${ }^{15}$ A publication by the International Commission of Radiological Protection reviewed experimental data about the in utero effect of radiation in animals and humans. They concluded that the risk of induction of malformation at low doses can be discounted. Data about the induction of severe mental retardation after irradiation from atomic bombs during the most sensitive prenatal period support a dose threshold of 6-31 rad between 8 and 15 weeks and 25-28 rad between 16 and 25 weeks. ${ }^{16}$ The data about loss of intelligence quotient after the bombing of Hiroshima and Nagasaki of 20-30 points per 100 rad exposure are more difficult to interpret. However, even in the absence of a true dose threshold, any effect on intelligence quotient at low doses would be undetectable.

The International Commission of Radiological Protection reported that the risk of induction of childhood solid tumours is similar to that of leukemia and that the risk of cancer in later life is similar to that following irradiation during childhood. In contrast, there are studies that suggest an increase in childhood cancer after in utero exposure to $1 \mathrm{rad}$. The excess absolute risk coefficient at this level of exposure is $6 \%$ per 100 rad. ${ }^{5}$ The British Oxford Survey of Childhood Cancer estimated the risk of cancer to be 0.022 per $100 \mathrm{rad}$. This is in agreement with the estimate of the Life Span Study from Japan that included survivors of the atomic bomb (0.028 per $100 \mathrm{rad}) .^{5}$

The American College of Obstetricians and Gynecologists has advised practitioners that, although there is no evidence that magnetic resonance imaging (MRI) is associated with adverse fetal effects, it should be avoided during the first trimester. However, because the fetal radiation dose is minimal, MRI should not be delayed if it is considered critical for the diagnosis of a serious maternal condition.

\section{Perceived risk of radiation}

Pregnant women who have undergone radiodiagnostic procedures have a high perception of teratogenic risk (perceived risk of $25.5 \%$ for major malformations). In contrast, women who have not undergone radiodiagnostic procedures have a lower perception of teratogenic risk (15.7\%). ${ }^{1}$ A questionnaire survey conducted in Israel reported that $40 \%$ family physicians $(n=86)$ and $70 \%$ of obstetricians $(n=20)$ would recommend therapeutic abortion to women who received radio-

\begin{tabular}{|c|c|}
\hline Test, area & Fetal dose, rad \\
\hline \multicolumn{2}{|l|}{ Radiograph } \\
\hline Upper extremity & $<0.001$ \\
\hline Lower extremity & $<0.001$ \\
\hline Upper gastrointestinal series (barium) & $0.048-0.360$ \\
\hline Cholecystography & $0.005-0.060$ \\
\hline Lumbar spine & $0.346-0.620$ \\
\hline Pelvis & $0.040-0.238$ \\
\hline Hip and femur series & $0.051-0.370$ \\
\hline Chest (2 views) & $<0.010$ \\
\hline Retropyelography & 0.800 \\
\hline Abdomen (kidneys, ureter and bladder) & $0.200-0.245$ \\
\hline Urography (intravenous pyelography) & $0.358-1.398$ \\
\hline Barium enema & $0.700-3.986$ \\
\hline \multicolumn{2}{|l|}{ CT scan } \\
\hline Head & $<0.050$ \\
\hline Chest & $0.100-0.450$ \\
\hline Abdomen (10 slices) & $0.240-2.600$ \\
\hline Abdomen and pelvis & 0.640 \\
\hline Pelvis & 0.730 \\
\hline Lumbar spine & 3.500 \\
\hline \multicolumn{2}{|l|}{ Other } \\
\hline Ventilation-perfusion scan & $0.06-1.00$ \\
\hline Potentially teratogenic dose & 5.00 \\
\hline
\end{tabular}

diagnostic procedures (intravenous pyelograms, abdominal radiography, barium enema) during early pregnancy. ${ }^{2}$

Similar trends have been reported in Canada. In a recent Canadian survey, 400 family physicians and 100 obstetricians were asked about their perception of the fetal risks associated with abdominal radiographs and CT scans during early pregnancy and whether they would recommend a therapeutic abortion following such exposure. ${ }^{3}$ Of the respondents, $40 \%$ of family physicians perceived the teratogenic risk associated with abdominal radiographs to be above baseline $(\geq 5 \%)$, and $61 \%$ estimated the risk associated with CT scans to be $5 \%$ or greater. Of obstetricians, $11 \%$ estimated the risk associated with radiographs to be $5 \%$ or greater, and $34 \%$ estimated the risk associated with $\mathrm{CT}$ scans to be $5 \%$ or greater. Among family physicians $1 \%$ would recommended an abortion if the patient had received a radiograph, and $6 \%$ would recommend an abortion after a CT scans. None of the obstetricians reported that they would recommend an abortion after a radiograph, but $5 \%$ would recommended an abortion after a CT scan during early pregnancy. ${ }^{3}$

The high perception by physicians of teratogenic risk associated with radiation could lead to unnecessary anxiety for pregnant women who have been inadvertently exposed and who seek counselling. It could also lead to delays in needed care for pregnant women. In one prospective study, 6 women $(10 \%$ of 
participants) exposed to low-dose diagnostic radiation during pregnancy chose to terminate the pregnancy, ${ }^{4}$ stating that it was because of anxiety often caused by physician's advice. Educational interventions about radiation exposure should be considered to facilitate accurate risk estimation by physicians.

\section{The cases revisited}

Case 1: You explain to your patient that the upper gastrointestinal series would have exposed her fetus to doses of radiation of $0.36 \mathrm{rad}$, or about 4 times the estimated dose of background environmental radiation that she would receive during the entire pregnancy. This is about 14-fold lower than the level that international radiation experts have suggested is the lower threshold for observable differences in pregnancy outcomes. You explain to your patient that many physicians and patients are unaware of the relatively high baseline risks for adverse outcomes in all pregnancies (with or without diagnostic x-ray exposure) for major malformations (3\%) and fetal growth restriction (4\%).

Case 2: It is critical that a pulmonary embolism be ruled out. A ventilation-perfusion scan would expose the fetus to radiation as low as 0.06-0.1 rad, which is well below the potentially teratogenic dose $(5 \mathrm{rad})$. A regular chest $\mathrm{CT}$ scan would expose the fetus to $0.45 \mathrm{rad}$, which is also well below the range of fetal risk. However, CT angiography is associated with higher doses and involves administration of a contrast agent. As her physician, your decision should be based on the individual operator and the diagnosis sensitivity and specificity of the unit.

Case 3: You advise your patient that a radiograph of the hip is associated with a fetal radiation dose of up to $0.37 \mathrm{rad}$. This is far below the fetal radiation dose considered as safe. Although this dose is not associated with reproductive risk, this procedure is not clearly indicated and will not affect treatment. The characteristics of your patient's pain suggest exercise-induced bursitis. Rest and analgesics are the treatment of choice, even if the diagnosis is osteoarthritis. At this time, the radiograph can be postponed while the patient is observed for any change.

\section{Conclusion}

The teratogenic effects of in utero exposure to ionizing radiation are dose-dependent with a well-defined threshold. There is no indication that radiodiagnostic doses of ionizing radiation during pregnancy increase the incidence of gross congenital malformations, intrauterine growth retardation or abortion.
The risks of such exposure are far below the spontaneous risks. The vast majority of radiodiagnostic procedures involve fetal radiation doses that are below the threshold of $5 \mathrm{rad}$. In cases of maternal exposure to ionizing radiation, fetal exposure should be estimated. Health care providers should be careful not to confuse maternal and fetal exposures because this may lead to erroneous decisions. The need for radiodiagnostic procedures during pregnancy should be carefully considered and the risks should be weighed against the benefits. A radiodiagnostic procedure should not be withheld from a pregnant woman if the procedure is clearly indicated and if it can affect her medical care. Unnecessary procedures (e.g., preemployment screen, routine periodic check-up) should be delayed because these procedures do not immediately contribute to patient's health care and might provoke anxiety.

This article has been peer reviewed.

Competing interests: None declared.

\section{REFERENCES}

1. Bentur Y, Norlatsch N, Koren G. Exposure to ionizing radiation during pregnancy: perception of teratogenic risk and outcome. Teratology 1991;43:109-12.

2. Fink D, Glick S. Misinformation among physicians about dangers of fetal x-ray exposure. Harefuah 1993;124:717-9.

3. Ratnapalan S, Bona N, Chandra K, et al. Physicians' perceptions of teratogenic risk associated with radiography and CT during early pregnancy. AJR Am J Roentgenol 2004;182:1107-9.

4. Cohen-Kerem R, Nulman I, Abramow-Newerly M, et al. Diagnostic radiation in pregnancy: perception versus true risks. J Obstet Gynaecol Can 2006;28:43-8.

5. Bentur Y. Ionizing and nonionizing radiation in pregnancy. In: Medication safety in pregnancy and breastfeeding. Philadelphia (PA): MacGraw Hill; 2007. p. 221-48.

6. National Council on Radiation Protection and Measurements (NCRP). Ionizing radiation exposure of the population of the United States: an update. Bethesda (MD): NCRP; 2008. Available: www.ncrponline.org/Docs_in_Review/NCRP0848.pdf (accessed 2008 Nov 4).

7. Brent RL. The effects of embryonic and fetal exposure to x-ray, microwaves, and ultrasound. Clin Perinatol 1986;13:615-48.

8. Center for Disease Control and Prevention (CDC). Prenatal radiation exposure: A fact sheet for physicians. Atlanta (GA): CDC; 2005. Available: http://emergency .cdc.gov/radiation/prenatalphysician.asp (available 2008 Nov 4).

9. Osei EK, Faulkner K. Fetal doses from radiological examinations. Br J Radiol 1999;72:773-80

10. Parry RA, Glaze SA, Archer BR. The AAPM/RSNA physics tutorial for residents: typical patient radiation doses in diagnostic radiology. Radiographics 1999;19:1289-302.

11. Dixon AK, Dendy P, Siral CT. How much does radiation dose matter? Lancet 1998;352:1082-3.

12. Sechopoulos I, Suryanarayanan, Vedantham S, et al. Radiation dose to organs and tissues from mammography: Monte Carlo and phantom study. Radiology 2008; 246: 434:43. Epub 2007 Dec 4.

13. Rehani MM, Berry M. Radiation doses in computed tomography. The increasing doses of radiation needed to be controlled. BMJ 2000;320:593-4.

14. Hujoel PP, Bollen AM, Noonan CJ, et al. Antepartum dental radiography and infant low birth weight. JAMA 2004;291:1987-93.

15. Brent RL. Commentary on JAMA article by Hujoel et al. Health Phys 2005;88:379-81. Comment on: JAMA 2004;291:1987-93.

16. Otake M, Schull WJ, Lee S. Threshold for radiation-related A-bomb survivors: a re- analysis. Int J Radiat Biol 1996;70:755-63. 